\title{
Formulation and Evaluation of Bisoprolol Hemifumarate Emulgel for Transdermal Drug Delivery
}

\author{
Rahman Gul, ${ }^{1,2^{*}}$ Yasmin Khan, ${ }^{3}$ and Tajala Aman ${ }^{4}$ \\ ${ }^{1}$ Faculty of Pharmacy \& Health Sciences, University of Balochistan, Pakistan. \\ ${ }^{2}$ Institute of Public Health, Quetta, Pakistan. \\ ${ }^{3}$ Sardar Bahadur Khan Women University, Quetta, Pakistan. \\ ${ }^{4}$ Quetta, Pakistan.
}

e-mail: gul.dotani@yahoo.com

\begin{abstract}
The aim of this study was to formulate and evaluate bisoprolol hemifumarate emulgel using carbopol 934P with the enhancers, thymus oil and olive oil. Thymus oil and olive oil were used as permeation enhancers. The emulsion formulations were added to a base gel. The emulgel was characterized for physical characteristics, stability, skin irritation, and drug release. Formulations F1 and F5 indicated the best in-vitro drug release when compared with other preparations. Bisoprolol hemifumarate emulgel containing carbopol 934P and thymus oil or olive oil enhancers could be further developed for a topical drug delivery system.
\end{abstract}

KEYWORDS: Emulgel, bisoprolol hemifumarate, topical drug delivery, carbopol, thymus oil, olive oil, dissolution

\section{INTRODUCTION}

diopathic premature ventricular contractions (PVCs) have been treated widely by $\beta$-blockers due to its safety profile and sympatholytic properties when compared to other anti-arrhythmic drugs. However, a number of studies have indicated that $\beta$-blockers do not do enough to curb idiopathic PVCs (1-3). In most situations, sympathetic creation could activate the incidence of idiopathic PVCs, but some studies have indicated that ventricular arrhythmias (VAs) build up as parasympathetic concerns are raised in a few patients (4). The heart rate reliance on idiopathic PVCs exposed to various autonomic phenomenon has been recommended (5). $\beta$-Blockers may have produced inadequate restraint of idiopathic PVCs because such existence of PVCs does not help by sympathetic creation. Along with $\beta$-blockers, bisoprolol hemifumarate has mainly selective $\beta 1$-blocking pharmacological action. In recent times, a transdermal $\beta$-blocker preparation containing bisoprolol has become medically accessible in Japan. Transdermal formulations of bisoprolol used for patients suffering from $d$ ysphasia as well as common conditions are not suitable $(6,7)$. Gels are a comparatively new group of drug delivery systems formed by entrapment of a huge quantity of hydroalcoholic or aqueous liquid within a system of colloidal solid particles that may be composed of inorganic substances, i.e., organic polymers or aluminium salts of synthetic or natural origin (8). This is due to an elevated aqueous constituent that releases dissolved drugs and enhances the easy movement of drugs via a medium that is basically a liquid compared to a cream or ointment base $(9,10)$, which is suitable for patients with certain conditions. There are a lot of benefits of gels as well as many drawbacks in the hydrophobic drugs delivery. However, to solve these restrictions, an emulgel may be formulated and utilized. Still, a hydrophobic healing moiety can benefit from the typical characteristics of gels (11). Emulsions and gels in dosage forms are termed "emulgel" (12). Currently, new polymers with composite actions as thickeners and emulsifiers are being used to formulate drugs. This is because the gelling ability of these constituents permits the preparation of established emulsions by declining interfacial and surface tension at a similar time, raising the thickness of the aqueous phase (13). Emulgel for dermatological applications has different encouraging characteristics, which are greaseless, emollient, thixotropic, easily spreadable, effortlessly removable, no staining, long shelf life, transparent, nice appearance, and bio-friendly (14).

The basic rationale of the present study was to formulate and analyze an emulgel preparation of bisoprolol 
hemifumarate, an antihypertensive drug, using carbopol 934P as a gelling polymer, with two kinds of permeation enhancers, i.e., thymus oil and olive oil. The effects of a gelling substance and permeation enhancers were assessed. Formulation $\mathrm{pH}$, spreading, viscosity, skin irritation, stability, and drug release were characterized.

\section{MATERIALS AND METHODS}

\section{Materials and Reagents}

Bisoprolol was given as a gift from Martindow Pvt. Ltd. (Quetta Factory, Pakistan). Carbopol 934P was provided by the University of Balochistan. Thymus oil and olive oil were obtained from the local market in Quetta. Dialysis cellulose membrane was obtained from Sigma Aldrich. All supplementary chemicals utilized were of standard grade.

\section{Emulgel Formulation}

The oil phases of the emulsion were formulated with span 20 in liquid paraffin. The aqueous phase was set with tween 20 mixed with distilled water. Propyl and methyl paraben preservatives were mixed with propylene glycol. Bisoprolol was mixed with ethanol, then the solution was dissolved in the aqueous phases. Olive and thymus oil were mixed separately in the oil phases. Both phases were heated to $70-80^{\circ} \mathrm{C}$. The oil phases were then poured into the aqueous phases with constant stirring and kept at room temperature.

Various formulations of the emulgel were prepared using different concentrations of carbopol permeation enhancers. The gel phase was prepared by dissolving carbopol 934P in distilled water and continuously stirring the mixture at a modest speed using a mechanical shaker while maintaining the $\mathrm{pH}$ at 5.5-6.0 using triethanolamine (TEA). The prepared gel was added into the emulsion in a 1:1 ratio and was mildly stirred to get emulgel (15). The concentrations of the various preparations are as shown in Table 1.

\section{Characterization of the Emulgel Physical Assessment}

The emulgel formulations were observed visually for their appearance, consistency, and color (16). The $\mathrm{pH}$ of all formulations was assessed with a calibrated $\mathrm{pH}$ meter (17). The viscosity of all the formulations was determined at room temperature $\left(25 \pm 1{ }^{\circ} \mathrm{C}\right)$ using a cone and flat-type viscometer (DV-III Ultra, Brookfield Engineering Laboratory, USA) (18). The emulgel samples were tested using an electrical conductivity meter (WTWcond197i, Weilhein, Germany) (19). Separation tests were determined for all formulations instantly after preparations. The centrifugation tests $5 \mathrm{~g}$ sample were carried out at $25^{\circ} \mathrm{C}$ for $10 \mathrm{~min}$ at $5000 \mathrm{rpm}$ (20).

Table 1. Composition of Emulgel Preparations

\begin{tabular}{|c|c|c|c|c|c|c|}
\hline Constituents (w/w) & F1 & F2 & F3 & F4 & F5 & F6 \\
\hline Bisoprolol & 1.0 & 1.0 & 1.0 & 1.0 & 1.0 & 1.0 \\
\hline Carbapol 934 & 1.0 & 1.0 & 1.0 & 1.0 & 1.0 & 1.0 \\
\hline Liquid paraffin & 7.5 & 7.5 & 7.5 & 7.5 & 7.5 & 7.5 \\
\hline Span 20 & 1.0 & 1.0 & 1.0 & 1.0 & 1.0 & 1.0 \\
\hline Propylene glycol & 5 & 5 & 5 & 5 & 5 & 5 \\
\hline Tween 20 & 0.5 & 0.5 & 0.5 & 0.5 & 0.5 & 0.5 \\
\hline Ethanol & 2.5 & 2.5 & 2.5 & 2.5 & 2.5 & 2.5 \\
\hline Propyl paraben & 0.03 & 0.03 & 0.03 & 0.03 & 0.03 & 0.03 \\
\hline Methyl paraben & 0.01 & 0.01 & 0.01 & 0.01 & 0.01 & 0.01 \\
\hline Thymus oil & 3.0 & 3.0 & 3.0 & - & - & - \\
\hline Olive oil & - & - & - & 3.0 & 3.0 & 3.0 \\
\hline Water & q.s & q.s & q.s & q.s & q.s & q.s \\
\hline
\end{tabular}

\section{Spreadability}

Emulgel spreadability was assessed in terms of diameter. A weighed $0.5 \mathrm{~g}$ emulgel sample of each formulation was applied on one glass slide of $10 \mathrm{~g}$ and the second glass slide of $10 \mathrm{~g}$ was covered with the first slide Table 2. As a result of firmness between the two slides, the increase in diameter of the circle in the formulations, the diameter was measured (21).

\section{Solubility Determination}

About $5 \mathrm{~mL}$ of buffer phosphate $(\mathrm{pH}$ 7.4) was taken in a test tube and added slowly into an excess amount of bisoprolol hemifumarate and shaken for $24 \mathrm{~h}$. The

Table 2. Physicochemical Parameters of Emulgel Batches

\begin{tabular}{|c|c|c|c|c|c|c|}
\hline Formulation & $\mathrm{pH}$ & Conductivity $(\mu \mathrm{S} / \mathrm{cm})$ & Phase Separation & Homogeneity & Spreadability $(\mathrm{cm})$ & Drug content $(\%)$ \\
\hline F1 & 5.9 & 0.2 & None & Best & 4.5 & 98.20 \\
\hline F2 & 5.8 & 0.1 & None & Best & 4.9 & 97.57 \\
\hline F3 & 5.6 & 0.0 & None & Best & 5.8 & 97.33 \\
\hline F4 & 5.7 & 0.2 & None & Best & 5.5 & 97.57 \\
\hline F5 & 5.9 & 0.0 & None & Best & 4.2 & 98.04 \\
\hline F6 & 5.8 & 0.1 & None & Best & 5.5 & 96.50 \\
\hline
\end{tabular}


supernatant solution was filtered via Whatman filter paper $(0.4 \mu \mathrm{m})$. The drug solution obtained was assessed using a UV-Visible spectrophotometer at $220 \mathrm{~nm}$.

\section{Skin Irritation Study}

The emulgel was applied to an area of rabbit skin, measuring about $6.0 \mathrm{~cm}^{2}$, and was covered by a gauze patch. This was fixed in contact with rabbit skin by way of semi-occlusive adhesive for $1 \mathrm{~h}$. The gauze was removed after $1 \mathrm{~h}$, and the remaining substance was removed, without damaging the skin. The skin was observed once a day for 7 days for signs of irritation (22).

\section{Drug Content Determination}

About $1 \mathrm{~g}$ of the prepared formulation was dissolved in $100 \mathrm{~mL}$ of $5 \%$ methanol solution and was energetically shaken for $1 \mathrm{~h}$, then sonicated for $15 \mathrm{~min}$, then centrifuged at $5000 \mathrm{rpm}$ for $30 \mathrm{~min}$. The solution was filtered via Whatman filter paper $(0.4 \mu \mathrm{m})$, using a validated highperformance liquid chromatography (HPLC) method to determine the drug content at $220 \mathrm{~nm}$. $(23,24)$.

\section{Assay and Purity Test of Bisoprolol Hemifumarate by Liquid Chromatography Determination \\ Reagents \\ Reagents included:}

- Bisoprolol hemifumarate

- Primary standard

- Acetonitrile for chromatography (LiChrosolv)

- Ammonium dihydrogen phosphate GR

- EMD 64 705: 1-\{4-[(2-isopropoxyethoxy) methoxymethyl]-phenoxy\}-3-isopropaol hemifumarate

- Benzyl alcohol: 4-\{2-hydroxy-3-iisopropylaminopropoxy)

- Acid (reference standard): 4-(2-hydroxy-3isopropylaminoproproxy)

- Aldehyde (benzaldehyde hemifumarate): 4-(2-Hydroxy-3-isopropylaminoproproxy)

- Hydroxyethyl ester: 2-hydroxyethyl ester hemifumarate

- Isopropyl ester

- Phenol: 4-(2-isopropoxyethoxymethyl)

- Ortho-phosphoric acid 85\% GR

\section{Reagent solutions}

A buffer solution with $\mathrm{pH} 2.5$ was prepared as follows. Approximately $1 \mathrm{~mL}$ of $85 \%$ ortho-phosphoric acid was added to $3.45 \mathrm{~g}$ of ammonium dihydrogen phosphate and dissolved in distilled water to make $1000 \mathrm{~mL}$. The buffer solution was used for the mobile phase, eluent A and B, and acetonitrile.

\section{Sample solution}

A sample solution was prepared as follows. In separate $100 \mathrm{~mL}$ volumetric flasks, $30 \mathrm{~mL}$ of the solvent (weighing $10 \mathrm{~g}$ ) was added to the emulgel sample (equivalent to approximately $100 \mathrm{mg}$ bisoprolol hemifumarate). The mixture was shaken mechanically for $1 \mathrm{~h}$ and was made up to $100 \mathrm{~mL}$ with $5 \%$ methanol solvent, then centrifuged.

\section{Standard solutions}

Accurately weighed $50 \mathrm{mg}$ of bisoprolol hemifumarate was dissolved in each solvent to make $50 \mathrm{~mL}$ of each standard solution including: EMD 64 705, benzyl alcohol, acid, aldehyde, hydroxyethyl ester, ester, isopropyl ester, and phenol.

Approximately $10 \mathrm{mg}$ aliquots of the substances were dissolved separately in a solvent to make 50 $\mathrm{mL}$ (ultrasound). About $5 \mathrm{~mL}$ of each solution was diluted to $100 \mathrm{~mL}$ with the solvent. The percentage of decomposition products is $1 \%$ relative to the active constituent (standard solution II). (The release control involves checking only for the two main decomposition products, ester and aldehyde).

\section{Chromatographic Conditions}

Apparatus Liquid chromatography with an automatic injection system

Detector UV-Vis spectrophotometer

Wavelength $220 \mathrm{~nm}$

Sample Volume $10 \mu \mathrm{L}$

Column Stainless steel cartridge LiChroCART 250-4 LiChrospher 60 RP-select B (5 um)

Oven

Temperature $\quad 50^{\circ} \mathrm{C}$

Flow rate $\quad 2 \mathrm{~mL} / \mathrm{min}$

\section{Dissolution Study}

Franz diffusion cells (Perm Gear, USA) were used for in vitro dissolution studies of bisoprolol hemifumarate emulgel via a cellulose membrane. The membranes were set among the donor and receptor holder of Franz diffusion cell apparatus. Receptor solvent was used as buffer phosphate ( $\mathrm{pH} 7.4)$, and $12 \mathrm{~mL}$ was filled into the receptor chamber. The sample chamber was filled with bisoprolol emulgel. The solvent temperature $\left(37^{\circ} \mathrm{C}\right)$ was 
maintained throughout the study. Approximately 1.0 $\mathrm{mL}$ samples were taken from each cell of the receptor medium at fixed time periods of $0.5,1.0,1.5,2.0,3.0$, 4.0, $8.0,12.0,16.0,20.0$, and $24.0 \mathrm{~h}$. Each cell was immediately filled with fresh medium $\left(37^{\circ} \mathrm{C}\right)$. The withdrawn samples were filtered, and the drug quantity was assessed by HPLC at $220 \mathrm{~nm}$ (25-27).

\section{Skin Permeation Study}

A skin permeation study of two formulations (F1, F5) was conducted in a Franz diffusion cell using excised skin from male rabbits weighing $2.5-3.0 \mathrm{~kg}$. Hair from the abdominal area was carefully shaved using a depilatory cream, and the skin was prepared. The dermal surface was carefully cleared of any blood vessels or adhering tissues and equilibrated for $1 \mathrm{hr}$ in buffer $\mathrm{pH} 7.4$ prior to the experiment. The rabbit's epidermis was fixed onto a Franz cell in a way that the stratum corneum part of the skin was constantly in contact with the transdermal emulgel in the donor section and the dermis side was constantly in contact with the receptor medium. The receptor section was filled with the medium at $37^{\circ} \mathrm{C}$. The receptor medium was magnetically stirred. A sample ( 1 $\mathrm{mL}$ ) was taken at various moments and quantified for drug constituents. The receptor solution was replaced with an equivalent volume of the phosphate buffer at each time period.

\section{Drug Release Kinetic Studies of Bisoprolol Hemifumarate}

A validated HPLC method was used for the analysis of drug release and the dissolution profile was computed. The linearity, regression analysis, and release characteristics for each formulation were observed. The correlation coefficient $\left(R^{2}\right)$ was analyzed for all the formulations. The release and permeability of the drug for each membrane was observed to follow a zero order, Higuchi, first order, Korsmeyer-Peppas, or Hixon-Crowell kinetic model. Calculations were conducted by DD Solver software. $(17,25)$.

\section{Stability Studies}

A stability study was performed on the formulated emulgel at various temperature for a period of 3 months. The emulgel samples were evaluated at $0 \pm 1{ }^{\circ} \mathrm{C}$ and 8 $\pm 0.1^{\circ} \mathrm{C}$ in a refrigerator, at $25 \pm 0.1{ }^{\circ} \mathrm{C}$ in the lab, and at $40 \pm 0.1{ }^{\circ} \mathrm{C}$ in an incubator for 15 days, then analyzed for physicochemical properties and drug content $(17,25)$.

\section{RESULTS AND DISCUSSION}

The bisoprolol emulgel was characterized for $\mathrm{pH}$, consistency, solubility, spreadability, homogeneity, and drug content, as given in Table 2.
No alterations were noted in its physical characteristics, including liquefaction, phase separation, color, and further characteristics like $\mathrm{pH}$. The $\mathrm{pH}$ was low, ranging from 5.6 to 5.9. This is in agreement with prior research in which topical dosage forms of flurbiprofen gel were formulated (22). Normal human skin has a $\mathrm{pH}$ ranging from 4.5 to 6 (23). Spreadability and consistency were examined over a time period of 90 days. Spreadability values varied from 4.2 to $5.8 \mathrm{~g} / \mathrm{cm} / \mathrm{s}$, which showed that the emulgel was spreadable with a minor shearing pressure. The emulgel had good solubility as well. The homogeneity was also good in all emulgel formulations without no lumps or visual particles.

\section{Rheological Measurement}

The rheological test was conducted for $10 \mathrm{~min}$ at 100 rpm. The results are shown in Figure 1.

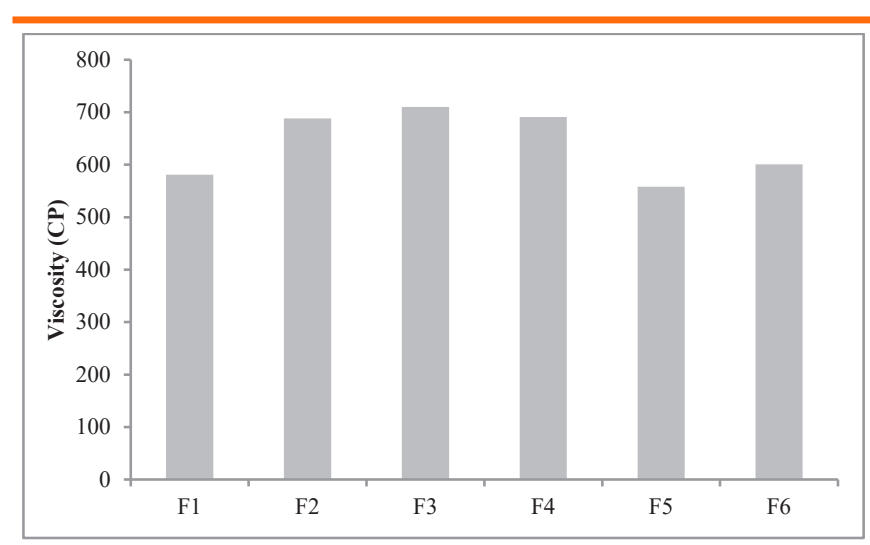

Figure 1. Viscosity of the Emulgel formulations F1-F6.

\section{Skin Irritation Study}

No symptoms of irritation (i.e., erythema or edema) were observed on the rabbit skin during the 7-day period. The skin did not show any sign of redness, lesion, or itching.

\section{Drug Content Determination}

The drug concentration of the bisoprolol emulgel was limited to 96.50 to $98.20 \%$. This shows a good uniformity. All the physical parameters of the formulations of emulgel prepared in this study indicate that the bisoprolol emulgels produced were suitable for transdermal applications.

\section{Dissolution and Permeation Studies}

Results of in vitro drug release and permeability studies are given in Table 3.

Drug release from the emulgel formulations ranged from $65 \%$ to $71 \%$ in descending order of $F 5>F 1>F 6>F 4>F 2$ $>F 3$, as shown in Figure 2. It was also observed that the essential oil increased drug diffusion, as indicated by (24).

The formulations with the highest release rates (F5 and F1) were further assessed for permeability through excised 
rabbit skin over a 24-h period. The results are shown in Figure 3. Permeation of $\mathrm{F} 5$ across the rabbit skin was $60 \%$ while $\mathrm{F} 1$, after adding the enhancers thymus oil and olive oil at $3.0 \%$, showed maximum penetration of $56 \%$.

The temperature $37^{\circ} \mathrm{C}$ also showed good drug release in this study. An increase in temperature from 32 to $37^{\circ} \mathrm{C}$ may also increase drug release/permeability, as shown in the case of acyclovir cream (28).

\section{Table 3. In Vitro Drug Release (\%) of Emulgel Formulations}

\begin{tabular}{|c|c|c|c|c|c|c|}
\hline $\begin{array}{c}\text { Time } \\
\text { (h) }\end{array}$ & F1 & F2 & F3 & F4 & F5 & F6 \\
\hline 0.5 & 2.381 & 2.481 & 2.181 & 2.081 & 2.881 & 2.181 \\
\hline 1 & 3.323 & 3.231 & 3.123 & 3.103 & 4.230 & 3.123 \\
\hline 2 & 5.921 & 6.321 & 5.000 & 5.021 & 7.421 & 6.021 \\
\hline 3 & 11.131 & 10.731 & 10.331 & 9.231 & 14.031 & 10.131 \\
\hline 4 & 15.765 & 15.165 & 14.065 & 12.265 & 17.565 & 15.065 \\
\hline 5 & 20.976 & 18.176 & 17.776 & 17.476 & 24.776 & 18.376 \\
\hline 6 & 22.312 & 20.771 & 19.012 & 20.702 & 28.012 & 20.122 \\
\hline 12 & 62.233 & 58.233 & 56.433 & 57.733 & 66.033 & 60.733 \\
\hline 24 & 70.143 & 65.743 & 65.043 & 66.643 & 70.763 & 67.163 \\
\hline
\end{tabular}

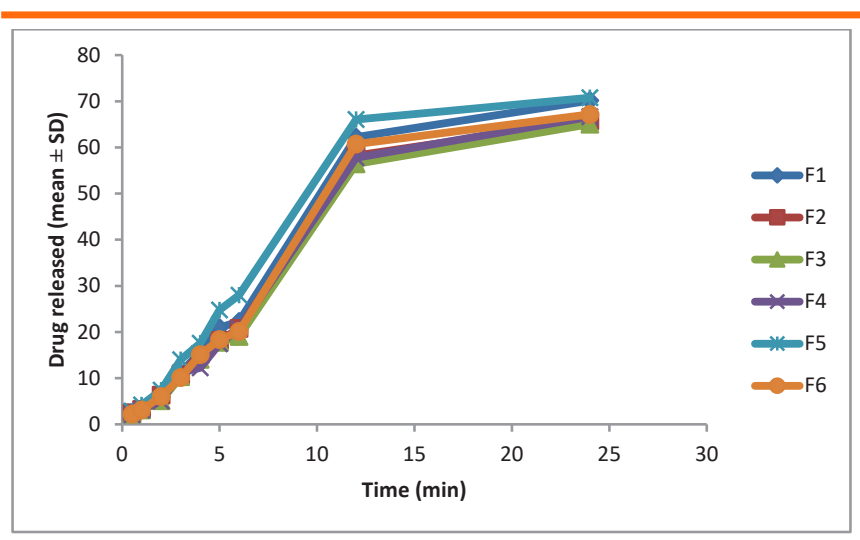

Figure 2. In vitro percentage drug release of Emulgel formulations F1-F6.

\section{Investigation of Drug Release Mechanism}

Results of kinetic modeling for drug release and permeability are given in Table 4.
There was not much difference in the release and permeability patterns of the drug for all formulations of bisoprolol hemifumarate emulgel. The release and permeability were best fit to First Order model.

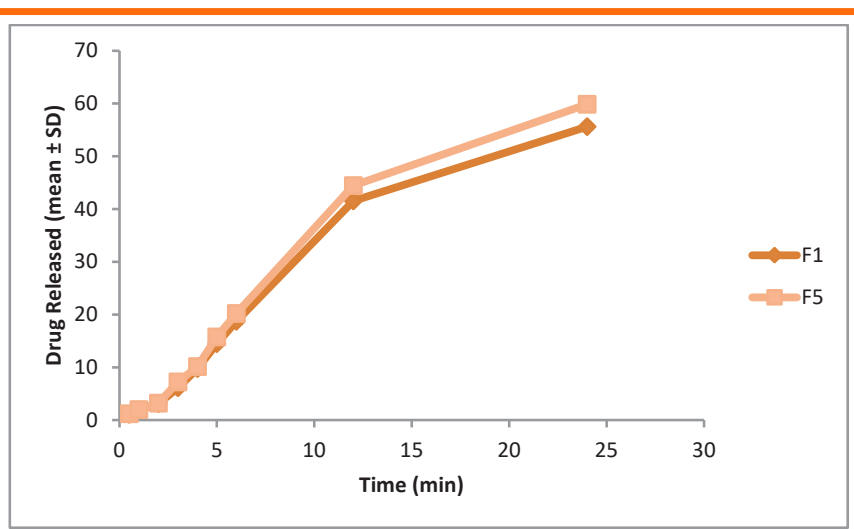

Figure 3. Ex vivo percentage release of Emulgel formulations F1 and F5.

\section{Stability Studies}

Stability studies of bisoprolol emulgel were found to be stable after 3 months of storage. There was no change in the color, $\mathrm{pH}$, phase separation, viscosity, and assay (drug content). In such a dosage form, formulation stability is the most important quality (22).

\section{CONCLUSION}

In this study, novel emulgel formulations for transdermal delivery of bisoprolol hemifumarate were developed. The formulations were physiochemically stable and topically non-irritable. Dissolution and skin permeability studies indicated that the emulgel formulations have potential to be novel delivery systems for bisoprolol. Bisoprolol hemifumarate emulgel containing carbopol 934P and thymus oil or olive oil enhancers could be further developed for a topical drug delivery system.

\section{ACKNOWLEDGMENT}

The authors thank Martindow, Quetta Factory, and the Faculty of Pharmacy and Health Sciences at the University of Balochistan for providing the chemicals and facilities; and the Institute of Public Health, Quetta, for the cooperation to complete this study.

Table 4. Kinetics Models of Emulgel Formulation Release $\left(R^{2}\right)$

\begin{tabular}{|c|c|c|c|c|c|c|}
\hline Formulation & Zero Order & First Order & Higuchi & Hixon-Crowell & Korsmeyer-Peppas & Best Fitting Model \\
\hline F1 & 0.8782 & 0.9400 & 0.7994 & 0.9394 & 0.9149 & First order \\
\hline F2 & 0.8793 & 0.9402 & 0.8001 & 0.9365 & 0.9158 & First order \\
\hline F3 & 0.8912 & 0.9396 & 0.7906 & 0.9382 & 0.9185 & First order \\
\hline F4 & 0.8930 & 0.9384 & 0.7860 & 0.9381 & 0.9177 & First order \\
\hline F5 & 0.8311 & 0.9441 & 0.8281 & 0.9342 & 0.9078 & First order \\
\hline F6 & 0.9738 & 0.9291 & 0.7837 & 0.9279 & 0.9049 & First order \\
\hline
\end{tabular}

$R^{2}=$ Coefficient of correlation . 


\section{CONFLICT OF INTEREST}

The authors disclosed no conflicts of interest related to this article.

\section{REFERENCES}

1. Krittayaphong, R.; Bhuripanyo, K.; Punlee, K.; Kangkagate, C.; Chaithiraphan, S. Effect of atenolol on symptomatic ventricular arrhythmia without structural heart disease: a randomized placebo-controlled study. Am. Heart J. 2002, 144, e10. DOI: 10.1067/mhj.2002.125516.

2. Ling, Z.; Liu, Z.; Su, L.; Zipunnikov, V.; Wu, J.; Du, H.; Woo, K.; Chen, S.; Zhong, B.; Lan, X.; Fan, J.; Xu, Y.; Chen, W.; Yin, Y.; Nazarian, $S$. Radiofrequency ablation versus antiarrhythmic medication for treatment of ventricular premature beats from the right ventricular outflow tract: prospective randomized study. Circ. Arrhythm. Electrophysiol. 2014, 7, 237-243. DOI: 10.1161/CIRCEP.113.000805.3. Yamada, T. Idiopathic ventricular arrhythmias: Relevance to the anatomy, diagnosis and treatment. J. Cardiol. 2016, 68, 463-471. DOI: 10.1016/j.jjcc.2016.06.001.

3. Hachiya, H.; Aonuma, K.; Yamauchi, Y.; Sekiguchi, Y.; lesaka, Y. Edrophonium-induced right ventricular outflow tract tachycardia. Pacing Clin. Electrophysiol. 2005, 28 (s1, Suppl. 1), S158-S162. DOI: 10.1111/j.1540-8159.2005.00007.x

4. He, W.; Lu, Z.; Bao, M.; Yu, L.; He, B.; Zhang, Y.; Hu, X.; Cui, B.; Huang, B.; Jiang, H. Autonomic involvement in idiopathic premature ventricular contractions. Clin. Res. Cardiol. 2013, 102, 361-370. DOI: 10.1007/s00392-013-0545-6.

5. Matsuoka, H.; Kuwajima, I.; Shimada, K.; Mitamura, H.; Saruta, T. Comparison of efficacy and safety between bisoprolol transdermal patch (TY-0201) and bisoprolol fumarate oral formulation in Japanese patients with grade I or II essential hypertension: randomized, double-blind, placebo-controlled study. J. Clin. Hypertens. (Greenwich) 2013, 15, 806-814. DOI: 10.1111/jch.12208.

6. Nakamura, K.; Inokuchi, R.; Hiruma, T.; Tokunaga, K.; Doi, K.; Nakajima, S. Switching therapy from intravenous beta blocker to bisoprolol transdermal patch for atrial fibrillation tachycardia. J. Anesth. 2016, 30, 891-894. DOI: 10.1007/s00540-016-2199-9.

7. Kumar, L.; Verma, R. In vitro evaluation of topical gel prepared using natural polymer. Int. J. Drug Deliv. 2010, 2, 58-63. DOI: 10.5138/ijdd.2010.0975.0215.02012.

8. Gennaro, A. R. Remington: The Science and Practice of Pharmacy, 19th ed. Mack Publishing Company, 1995.

9. Ansel, H. C.; Allen, L. V., Jr.; Popovich, N. G. Pharmaceutical Dosage Forms and Drug Delivery Systems, 7th ed. Lippincott Williams and Wilkins, New York, 1999.

10. Steiger, M. Topical emulsion- gel composition comprising diclofenac sodium. Australia Patent no. AU2003264086A1. August 21, 2003.

11. Mohamed, M. I. Optimization of chlorphenesin emulgel formulation. AAPS J. 2004, 6, e26. DOI: 10.1208/ aapsj060326.

12. Gupta, A.; Mishra, A. K.; Singh, A. K.; Gupta, V.; Bansal, P. Formulation and evaluation of topical gel of diclofenac sodium using different polymers. Drug Invention Today 2010, 2, 250-253.

13. Stanos, S. P. Topical agents for the management of musculoskeletal pain. J. Pain Sympt. Manage. 2007, 33, 345-355. DOI: 10.1016/j.jpainsymman.2006.11.005.

14. Kasliwal, N.; Derle, D.; Negi, J.; Gohil, J. Effect of permeation enhancers on the release and permeation kinetics of meloxicam gel formulations through rat skin. Asian J. Pharm. Sci. 2008, 3, 193-199.

15. Jain, A.; Deveda, P.; Vyas, N.; Chauhan, J. Development of antifungal emulsion based gel for topical fungal infection(s). IJPRD 2011, (3).

16. Gul, R.; Jan, U. S.; Ahmad, M.; Akhtar, M.; Faridullah, S. Formulation, characterization, in vitro and ex vivo release of Ephedra extract from topical preparations using dialysis membrane and rabbit skin. Dissolut. Technol. 2017, 24, 2430. DOI: 10.14227/DT240417P24.

17. Kim, J. Y.; Song, J. Y.; Lee, E. J.; Park, S. K. Rheological properties and microstructures of Carbopol gel network system. Colloid Polymer Sci. 2003, 281, 614-623.

18. Djordjevic, L.; Primorac, M.; Stupar, M. In vitro release of diclofenac diethylamine from caprylocaproyl macrogolglycerides based micro emulsions. Int. J. Pharm. 2005, 296, 73-79. DOI: 10.1016/j.ijpharm.2005.02.014.

19. Ueda, C. T.; Shah, V. P.; Derdzinski, K.; Ewing, G.; Flynn, G.; Maibach, H.; Marques, M.; Rytting, H.; Shaw, S.; Thakker, K.; Yacobi, A. Topical and transdermal drug products. Pharm. Forum. 2009, 35, 750-759.

20. Desai, K. G. H. Enhanced skin permeation of rofecoxib using topical microemulsion gel. Drug Devel. Res. 2004, 63, 33-40. DOI: 10.1002/ddr.10386.

21. Parkash, R.; Rao, R. J. Formulation and evaluation of herbal gel containing lantana camara leaves extract. Pharm. Clin. Res. 2010, 3, 126-129.

22. Gul, R.; Jan, S. U.; Khan, A.; Jahan, N.; Rahman, R.; Sherani, S.; Tariq, N. Effect of thyme oil on the transdermal permeation pseudoephedrine $\mathrm{HCl}$ from topical gel. Dissolut. Technol. 2019, 26, 18-23. DOI: 10.14227/DT260419P18.

23. Mutalik, N. U.I Udupa, N. Glibenclamide transdermal patches: Physicochemical, pharmacodynamic, and pharmacokinetic evaluations. J. Pharm. Sci. 2004, 93, 1577-1594. DOI: 10.1002/jps.20058.

24. Iyer, B. V.; Vasavada, R. C. Evaluation of lanolin alcohol films and kinetics of triamcinolone acetonide release. J. Pharm. Sci. 1979, 68, 782-787. DOI: 10.1002/jps.2600680631.

25. Sintov, A. C.; Krymberk, I.; Gavrilov, V.; Gorodischer, R. Transdermal delivery of paracetamol for paediatric use: effects of vehicle formulations on the percutaneous 
penetration. J. Pharm. Pharmacol. 2003, 55, 911-919. DOI: 10.1211/0022357021486.

26. Gokhale, R.; Schmidt, C.; Alcorn, L.; Stolzenbach, J.; Schoenhard, G.; Farhadieh, B.; Needham, T. Transdermal drug delivery systems of albuterol: in vitro and in vivo studies. J. Pharm. Sci. 1992, 81, 996-999. DOI: 10.1002/ jps.2600811007.

27. Kulkarni, M.; Potdar, S.; Date, A. A.; Marfatiya, A. In vitro release testing of Acyclovir topical formulations using immersion cells. ASSAY Drug Devel. Technol. 2021, 19, 7584. DOI: 10.1089/adt.2020.995. 